\title{
License Plate Identification in Complex Background Videos
}

\author{
Aparna M \\ KMCT College of Engineering and Technology, Calicut, Kerala, India
}

\begin{abstract}
Object segmentation is the process of abstraction of object from an image or video frame. There are several techniques available to handle object segmentation from video frames. Existing methods have mainly two problems such as they do not consider the spatial parameters of an object in a frame, and it is not possible to handle complex scenes with highly dynamic background movements with existing methods. With varying scales, a robust analysis mechanism is required to handle background regions or foreground movements. This paper proposes a solution to these problems and provides an efficient algorithm to overcome these problems and advanced features like object identification also included. Main objective of the paper is number plate or license plate identification from complex background videos using the technique Optical Character Recognition.
\end{abstract}

Keywords: Background Subtraction, Law Rank Matrix, ObjectSegmentation, Object Identification, Optical Character Recognition, Robust Principal Component Analysis

\section{Introduction}

Background subtraction or Foreground Detection, is a technique in the fields of image processing and computer vision wherein an image's foreground is extracted for further processing. Also it is a method used to detect moving parts bysubtracting them from the established background. Principal component analysis is a technique used for dimensionality reduction. A new frame was projected onto the subspace spanned by the trained principle components, and the residues indicate the presence of new foreground objects. A more advanced method is robust Principal component analysis. It is proposed to use an 11 -norm to constrain the foreground matrix because these regions must be a sparse matrix with a small fraction of nonzero entries. It is also assumed that the background images are linearly correlated with each other, forming a low-rank matrix L. The methods now exist are based on the RPCA method.

Another concept in background subtraction is sparse signal recovery. It provides a framework to deal with various problems in machine learning and signal processing, also find low complexity methods with acceptable performance. With these RPCA and sparse signal recovery, concept of low rank and group sparse RPCA are used for object recognition from the video.

Identification of objects from different videos provide a better analysis for the video provided they are very complex in nature. The LDA (Linear Discriminant Analysis) classifier and a database is used for the process of object identification. LDA frequently achieves good performances in the tasks of face and object recognition, even though the assumptions of common covariance matrix among groups and normality are often violated. It also commonly used for dimentionality reduction, so that the segmentation of objects and the feature extraction got better performance than other classifiers like PCA and SVM. The features are compared with the databae and the object is identifired.

LPR (License Plate Recognition) is an image-processing technology used to identify vehicles by their license plates.
This technology is used in various security and traffic applications, such as the access-control system.LPR is also known as Automatic Vehicle Identification (AVI),Car Plate Recognition (CPR),Automatic Number Plate Recognition (ANPR),Car Plate Reader (CPR),Optical Character Recognition (OCR) for Cars. The license Plate can be identified in a complex background video using the technique Optical Character Recogotion.OCR is a method in computer science used for the conversion or recognition of texts or characters in any situations.

This paper proposes a method to identify the number plate of moving vehicle in complex background videos.section 2 is the detailed expalanation of background subtraction based on Low-rank and Structred Sparser Decomposition. Section 3 deals with Object Identification and Section 4 giving the details about how License Plate Number can be identified in complex background videos. Section 5 is the implimentaion results and section 6 include conclusion and future scope.

\section{Background Subtraction Based on Low- rank and Structured Sparse Decomposition}

A method called Low-rank And Structured Sparsity Decomposition (LSD) For Foreground Detection and Group-Sparse RPCA method are used to solve the above problems such as considering about spatial parameters and complex dynamic backgrounds. The concept of RPCA is used to take into account the methods of low rank and sparsity matrix.11 norm and regularizing parameter[4] are used to prove the method. In this a technique called Low-rank and Structured Sparsity Decomposition (LSD)ForForeground Detection is used to for modeling sparse outliers by Structured sparsity-inducing norms. The core concept is that the '11-norm treats each pixel independently and the structured sparsity norm can take into account possible relations among subsets of the entries. Systematic steps are there to process the proposed method, after all Optimization Methods like Augmented Lagrange Multiplier (ALM) method are used to optimize the result. Frame work of the proposed method can be divided into three. 


\section{International Journal of Science and Research (IJSR) \\ ISSN (Online): 2319-7064}

Index Copernicus Value (2013): 6.14 | Impact Factor (2015): 6.391

\subsection{Decomposition via LSD}

A structured sparsity based RPCA scheme can better estimate background. It is also sensitive to some dynamic background motions. The obtained candidate groups denote both foreground objects and a few background motions.

\subsection{Motion Saliency Check}

Background motion is usually smaller and more regular than foreground object motion. So the foreground object will form a distinct trajectory from the background in a temporal slice on planes. The analysis of temporal slices will detect and generate a motion saliency map.Calculate each group's average saliency from the motion saliency map. From these values, setting threshold value to eliminate small groups and small motions

\subsection{Group Sparse RPCA}

Group-sparse RPCA is used to carry out the final foreground detection from those motion saliency groups. on-stationary background motions are filtered out by adjusting the value of $\lambda$ and get the foreground object.

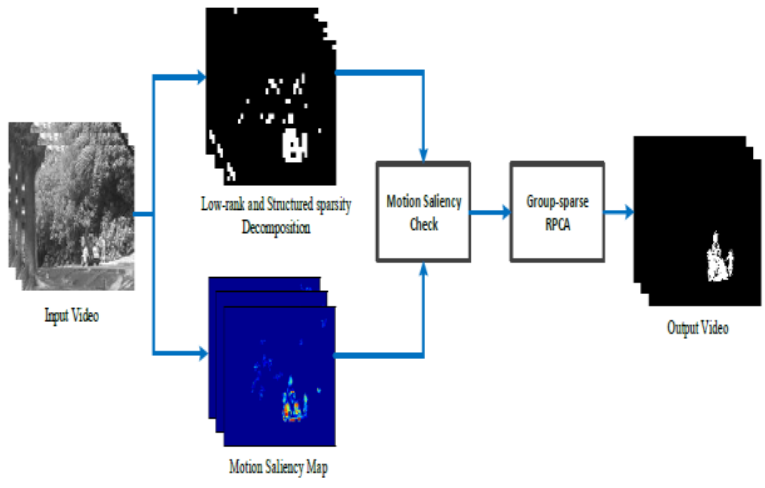

Figure 1: Framework for the proposed method

\section{Object Identification}

Detected objects can be identified with the help of a classifier LDA (Linear Discriminant Analysis) and a database. The LDA) classifier and a database is used for the process of object identification.LDA frequently achieves good performances in the tasks of face and object recognition, even though the assumptions of common covariance matrix among groups and normality are often violated. It also commonly used for dimensionality reduction, so that the segmentation of objects can be done easier. This extracted features based on the LDA algorithm can be compared with already trained dataset. The dataset include different samples of objects (mainly vehicles) so that the comparison can be done. The algorithm for LDA classifier is given in Algorithm 1.

\section{Algorithm 1 :LDA Classifier}

Input: Pixels of video frame

Output: Objects in the video

1) Compute the $d$-dimensional mean vectors for the different classes from the dataset.
2) Compute the scatter matrices (between-class and withinclass scatter matrix).

3) Compute the eigenvectors $\left(\mathbf{e}_{1}, \mathbf{e}_{2}, \ldots, \mathbf{e}_{\mathbf{d}}\right)$ and corresponding eigenvalues $\left(\lambda_{1}, \lambda_{2}, \ldots, \lambda_{d}\right)$ for the scatter matrices.

4) Sort the eigenvectors by decreasing eigenvalues and choose $\mathbf{k}$ eigenvectors with the largest eigenvalues to form a $d \times k$-dimensional matrix $\mathbf{W}$ (where every column represents an eigenvector).

5) Use this $d \times k$ eigenvector matrix to transform the samples onto the new subspace. This can be summarized by the equation $\boldsymbol{Y}=\boldsymbol{X} \times \boldsymbol{W}$ (where $\boldsymbol{X}$ is an $n \times d$-dimensional matrix; the $i$ th row represents the $i$ th sample, and $\boldsymbol{Y}$ is the transformed $n \times k$-dimensional matrix with the $n$ samples projected into the new subspace).

After the working of Algorithm 1,an Eigen vector matrix will get. This includes the Eigen values which represent the features of objects. These Eigen values can be compared with the dataset and identify the object.Figure 2 shows the result of object identification.

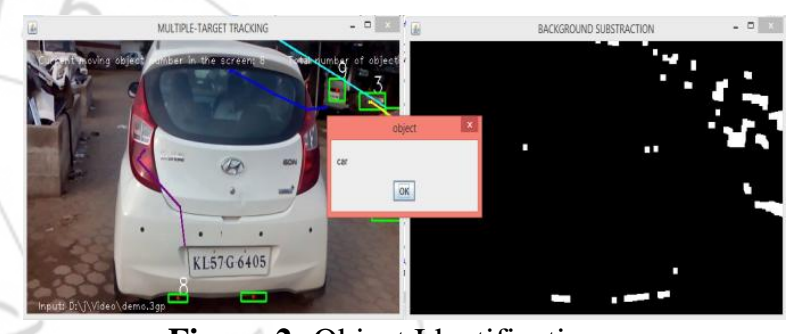

Figure 2: Object Identification

\section{License Plate Identification}

LPI (License Plate Identification) is an image-processing technology used to identify vehicles by their license plates/number plates. This field of computer science mainly deals with the security system and traffic control system. The system uses illumination and a camera to take the image of the front or rear of the vehicle, then an image-processing software analyzes the images and extracts the plate information. This data is used for enforcement, data collection, and can be used to open a gate if the car is authorized or keep a time record on the entry or exit for automatic payment calculations. Main advantage of the system is that the system can keep an image record of the vehicle which is useful in order to fight crime. Early LPI systems sufferred from a low recognition rate, lower than required by practical systems. The external effects (sun and headlights, bad plates, wide number of plates types) and the limited level of the recognition software and vision hardware yielded low quality systems. However, recent improvements in the software and hardware have made the LPR systems much more reliable and wide spread.

The problem with recognition of number plates from images is that, there is no guarantee for the images of moving vehicles in the road, There is no proper method to identify the license plate from the cctv recorded video. Here suggested a method to identify the number plate from videos even if they include complex background. Optical Character Recognition method is used for the recognition, which is a better method than other existing methods.It will identify the 


\section{International Journal of Science and Research (IJSR) \\ ISSN (Online): 2319-7064 \\ Index Copernicus Value (2013): 6.14 | Impact Factor (2015): 6.391}

number plate even if the number plate is in different color or format. Working algorithm for OCR is given in Algorithm 2.

\section{Algorithm 2: Optical Character Recognition}

\section{Input: video frame}

Output: Number Plate of the detected object.

1) A low resolution video can be used as the input for the proposed system. The input video may contain several objects and it may include complex background motion.

2) The given low resolution input video can be divided into a number of frames based on the given condition. These frames are read by the system and desired functions are performed based on the features of these frames.

3) This step is a major task in the proposed project. Objects in the given input video can be detectedbased on a new method called Low rank and structured sparsity Decomposition (LSD).The relevant objects are detected in comparison with the background motion.

4) The background can be subtracted to abstract the foreground only.

5) The detected objects in the video can be identified with a predefined database, in which it contains several examples of objects. In comparison with this database and detected object, system canidentify the object.

6) If the identified object is not a vehicle, then it display its name in comparison with the database.

7) This step also a major task in the proposed system. If the identified object is a vehicle (mainly fourwheelers), then it goes to license plate identification based on Optical Character Recognition(OCR).

8) The identified license plate number is displayed.

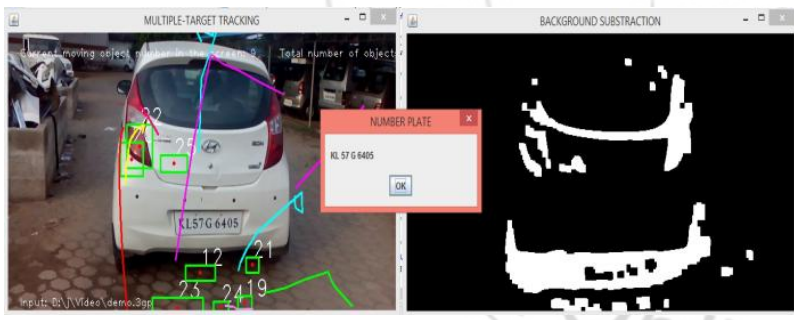

Figure 3: LPI

License plate of the detected vehicle object in the input video can be displayed based on Algorithm 2.Figure 3 shows the result of License Plate Identification.

\section{Implementation Results}

Input videos for the system can be low resolution videos, so that the detection of objects can be easier. Object detection, Object Identification, License Plate Identification can be implemented using java language with the support of OpenCV library in Eclipse mars 0.1 IDE.

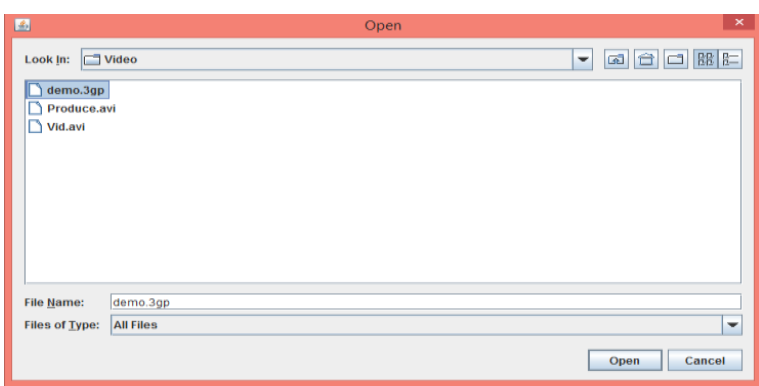

Figure 4: Selection of demo video

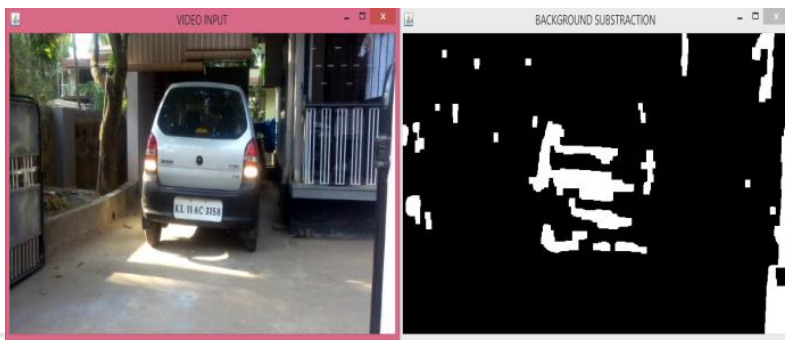

Figure 5: Background Subtraction

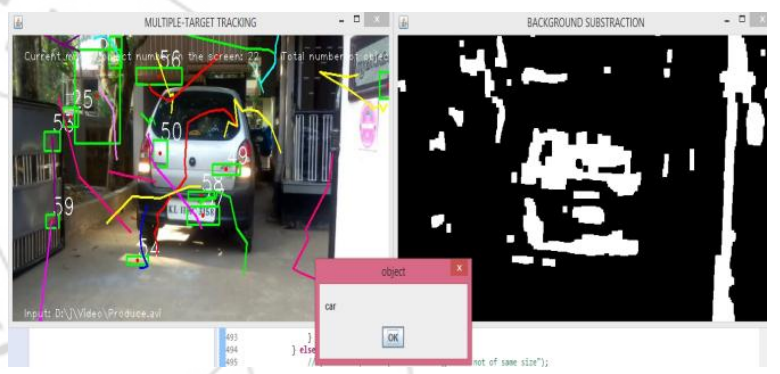

Figure 6: Object Identification

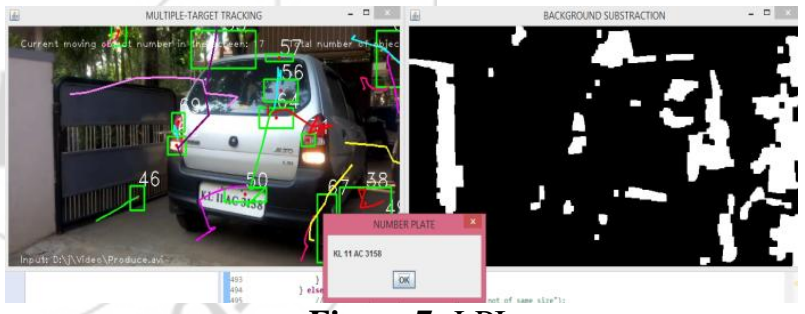

Figure 7: LPI

There are some difficulties with the combination of java with Open CV. Creating an interface between the user and the software form a white screen as the output. So direct seletion of input video can be done. Only trained input video can only run in the system. videos include limited number of objects, which are vehicles. even if these difficulties are existing, the system outperform with the existing methods, which are handling only the images.

\section{Conclusion and Future Scope}

Several aspects on background subtraction or foreground detection are considered. There are some problems with the already existing methods so that a new algorithm called LSD is proposed. A low-rank and structured-sparse matrix decomposition method also was proposed to take into account the spatial connection of the foreground regions and a group sparse RPCA method was proposed to ensure that the system is able to tolerate dynamic background variations to detect real foreground objects. Object Identification and Licence Plate identification in complex background videos 


\section{International Journal of Science and Research (IJSR) \\ ISSN (Online): 2319-7064}

Index Copernicus Value (2013): 6.14 | Impact Factor (2015): 6.391

are also included to extend the concept of object segementation.LDA classifier and OCR methods are used for identification of objects and LPI.

The system is efficient and provide accurate result than existing methods. The main application areas are Medical field, Object detection, Traffic control system etc. It may be possible to elaborate the paper in case of real time requirements. It may be possible to modify the method to handle more noisy frames. Possible to elaborate the method to detect a particular object only in a frame.

\section{References}

[1] Emmanuel J. Cand_es,Xiaodong Li, Yi Ma, and John Wright," Robust Principal Component Analysis?", December 17, 2009.

[2] Yawen Xue,Xiaojie Guoand Xiaochun Cao," Motion Saliency Detection Using Low-Rank And Sparse Decomposition ”, School of Computer Software, Tianjin University, Tianjin 300072, China

[3] Zhi Gao, Loong-Fah Cheong, and Mo Shan," Blocksparse RPCA for Consistent Foreground Detection", ECE Department, National University of Singapore, Singapore.

[4] Julien Mairal, Rodolphe Jenatton, Guillaume Obozinski," Network Flow Algorithms for Structured Sparsity", INRIA/ENS/CNRS UMR 8548.

[5] Kui Jia, Tsung-Han Chan, and Yi Ma," Robust and Practical Face Recognition via Structured Sparsity", ONR N00014-09-1-0230, NSF CCF 09-64215, NSF IIS 11-16012, andDARPA KECoM 10036- 100471.

[6] Xiaodi Hou and Liqing Zhang," Saliency Detection: A Spectral Residual Approach" , 1-4244-11807/07/\$25.00 @2007 IEEE.

[7] Cui, Qingshan Liu, Dimitris Metaxas," Temporal Spectral Residual: Fast Motion SaliencyDetection", MM'09, October 19-24, 2009, Beijing, China

[8] Liyuan Li, Weimin Huang, Irene Y.H. Gu, Qi Tian," Statistical Modeling of Complex Backgroundsfor Foreground Object Detection", IEEE TRANSACTIONS ON IMAGE PROCESSING, VOL. 13, NO. 11, NOVEMBER 2004.

[9] Makito Seki,Toshikazu Wada,Hideto Fujiwara, Kazuhiko Sumi," Background Subtraction based on Cooccurrence of Image Variations", 1063-6919/03 $\$ 17.00$ @ 2003 IEEE

[10] Liyuan Li, Weimin Huang, Irene Y.H. Gu, Qi Tian,” Foreground Object Detection from Videos ContainingComplex Background", November 2-8, 2003, Berkeley, California, USA.

[11] Xinyi Cui, Junzhou Huang, Shaoting Zhang, and Dimitris N. Metaxas," Background Subtraction Using Low Rank and Group Sparsity Constraints", ECCV 2012, Part I, LNCS 7572, pp. 612-625, 2012.

[12] Xin Liu, Guoying Zhao,Jiawen Yao, and Chun Qi," Background Subtraction Based on Low-rank and Structured Sparse Decomposition", 10.1109/TIP.2015.2419084, IEEE Transactions on Image Processing

[13] Micheal E tipping and Christopher M Bishop,'Probabilistic Principal Component
Analysis",journel of the Royal Statistical Research society,Series B 61 part 3.

[14] Aparna M, Suhara S," Various Aspects On Object Segmentation From Complex Background Videos", ISSN: 2277-9655,IJSERT.

\section{Author Profile}

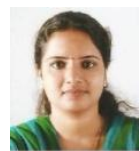

Aparna $\mathbf{M}$ is pursuing her $\mathbf{M}$.Tech degree in Computer Science and Engineering from KMCT College of Engineering, Calicut University. She obtained her B.Tech Degree in Computer Science and Engineering from Cochin University of Science And Technology, in 2013.

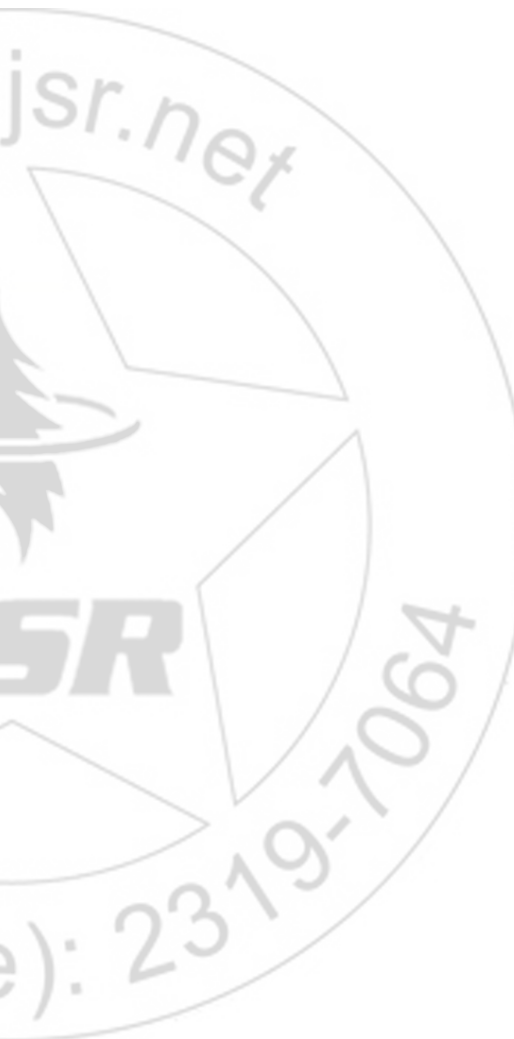

(1)

